\title{
Soluzioni periodiche di una classe di sistemi di tre equazioni differenziali ordinarie (*).
}

\author{
Gamtano Villari (a Firenze)
}

\begin{abstract}
Sommario. - Si studia il fenomeno della persistenza di soluzioni periodiche per una classe di sistemi di tre equazioni differenziali ordinarie soggetti a particolari perturbaeioni, e si stabilisce un criterio di esistenza di soluzioni periodiche che estende un analogo risultato valido per equazioni del terz' ordine.
\end{abstract}

1. - In un precedente lavoro [6] ( $\left.{ }^{1}\right)$ si è studiato l'effetto che una perturbazione periodica ha su equanioni lineari autonome del terzo ordine, ai fini dell'esistenza di soluzioni aventi lo stesso periodo della perturbazione.

E ben noto che un'equazione del terzo ordine può sempre ridursi ad un sistema di tre equazioni del primo ordine. Tuttavia talune applicazioni (in particolare nel campo della regolazione automatica) impongono lo studio di sistemi di tre (o più) equazioni che non sono riducibili ad una singola equazione di ordine 3 (o superiore), e cio in relazione allo studio della sta. bilità ed a quello dell' esistenza di soluzioni periodiche (cfr. [1], [2], [3]).

Tale considerazione induce quindi a ricercare possibili estensioni ai sistemi dei risultati conseguiti per le equazioni di ordine superiore al primo.

Nella presente ricerca abbiamo studiato il caso di un sitema di tre equazioni una delle quali sia soggetta ad una perturbazione periodica somma di un termine limitato e di nno dipendente dalla sola variabile che compare in derivazione nella equazione perturbata.

L'uso del metodo di LERAY-SOHAUDER conduce a stabilire un criterio di esistenza di soluzioni periodiche che non implica alcuna particolare limitazione sulla perturbazione suddetta ed estende un risultato ottenuto in [6] per una classe di equazioni del terz' ordine.

Detto risulfato ha essenzialmente carattere esemplificativo. in quanto il metodo usato consente di affermare l'esistenza di soluzioni periodiche anche in relazione a perturbazioni di tipo diverso da quello considerato, dando luogo ad una vasta casistica che verrà approfondita in dettaglio altrove.

(*) Ricerca eseguita nell'ambito del Gruppo di Ricerca n. 11 del C.N.R.

(1) I numeri in parentesi quadra si riferiscono all'elenco bibliografico posto alla fine del lavoro. 
2. - Si consideri il sistema

$$
\left\{\begin{array}{l}
\dot{x}=a_{11} x+a_{12} y+a_{13} z \\
\dot{y}=a_{21} x+a_{22} y+a_{23} z \\
\dot{z}=a_{31} x+a_{32} y+a_{33} z+p(t, x, y, z)+f(z)
\end{array}\right.
$$

ove $\cdot=d / d t$, le $a_{i j}(i, j=1,2,3)$ sono costanti reali, $p(t, x, y, z)$ e $f(z)$ sono funzioni continue dei rispettivi argomenti, ovunque definite,

$$
|p(t, x, y, z)| \leq p<+\infty
$$

ed esista un numero $\omega>0$. tale che

$$
p(t+\omega, x, y, z)=p(t, x, y, z)
$$

per ogni $t$ e qualunque sia la terna $x, y$, $z$.

Indicheremo con $A$ la matrice $\left(a_{i j}\right)$ e con $A_{i j}(i, j=1,2,3)$ il complemento algebrico dell' elemento $a_{i j}$ in $A$.

Supporremo anche che:

$$
\begin{aligned}
& \text { sia soddisfatta almeno una delle due condizioni } \\
& \qquad A_{33} \neq 0, \quad a_{18} A_{1^{3}}+a_{23} A_{23} \neq 0,
\end{aligned}
$$

e che inoltre si abbia

$$
a_{13} a_{31}+a_{2 s} a_{32}>0
$$

Cominciamo con l'osservare che, per le ipotesi (4) e (5), è possibile sce. gliere il numero $\bar{a}_{38}$ in modo che il sistema omogeneo

$$
\left\{\begin{array}{l}
\dot{x}=a_{11} x+a_{12} y+a_{13} z \\
\dot{y}=a_{21} x+a_{22} y+a_{28} z \\
\dot{z}=a_{31} x+a_{32} y+\overline{a_{33} z}
\end{array}\right.
$$

risulti privo di solnzioni periodiche diverse dalla nulla.

Tale condizione sarà soddisfatta se, e soltanto se, non esistono radici con 
parte reale nulla per l'equazione caratteristica del sistema (6)

$$
q^{3}+a q^{2}+b q+c=0
$$

ove si è posto

$$
\begin{aligned}
& a=-\operatorname{tr} \bar{A}=-\left(a_{11}+a_{22}+\bar{a}_{88}\right), \\
& b=\bar{A}_{11}+\bar{A}_{22}+A_{33}, \\
& c=-\operatorname{det} \bar{A}=-\left(a_{13} A_{13}+a_{28} A_{23}+\bar{a}_{33} A_{33}\right)\left({ }^{2}\right) .
\end{aligned}
$$

D'altra parte è ben noto che l'esistenza di radici con parte reale nulla della (7) implica $c=0$, oppure, nel caso $c \neq 0$, il contemporaneo verificarsi delle relazioni

$$
b>0, \quad a c>0, \quad a b-c=0
$$

pertanto la proprietà in oggetto risulterà dimostrata quando si provi che, con opportuna scelta di $\bar{a}_{33}$, si ha $c \neq 0$ e che una almeno delle (8) non è soddisfatta.

La condizione $c \neq 0$ segue subito dalla ipotesi (4), e ciò indipendentemente dalla scelta di $\bar{a}_{33}$ nel campo reale, fatta eccezione (nel caso $A_{33} \neq 0$ ) per il numero $-\left(a_{13} A_{13}+a_{23} A_{23}\right) / A_{33}$.

Inoltre la (5) può scriversi nella forma

$$
-\left(\overline{A_{11}}+\bar{A}_{22}+A_{33}\right)+\left(a_{11}+a_{22}\right) \bar{a}_{33}+A_{33}>0 .
$$

Pertanto, se $a_{11}+a_{22} \neq 0$, scegliendo $\bar{a}_{83}$ in modo che sia

$$
\left(a_{11}+a_{22}\right) \bar{a}_{83}>-A_{83}
$$

dalla (9) si ha

$$
\bar{A}_{11}+\bar{A}_{22}+A_{33}=b<0
$$

e non sarebbe soddisfatta la prima delle (8).

Se $a_{11}+a_{22}=0$, e $a_{13} A_{13}+a_{23} A_{23} \neq 0$, si può sempre pensare di scegliere

(2) Indichiamo con $\vec{A}$ la matrice ottenuta sostituendo in $A$ il numero $a_{33}$ con $\bar{a}_{33}$, e con $\bar{A}_{i j}$ i complementi algebrici dei suoi elementi quando non coincidono con quelli degli elementi corrispondenti di $A$. 
$\bar{a}_{33}$ in modo che risultino verificate le condizioni

$$
\begin{gathered}
\bar{a}_{33}\left(a_{13} A_{13}+a_{23} A_{23}\right)<0, \\
\left|\bar{a}_{33} A_{23}\right|<\left|a_{13} A_{13}+a_{23} A_{23}\right|,
\end{gathered}
$$

e quindi, avendosi in tal caso

$$
\begin{gathered}
a=-\bar{a}_{33}, \\
\operatorname{sign} c=-\operatorname{sign}\left(a_{13} A_{13}+a_{23} A_{23}\right),
\end{gathered}
$$

non sarebbe soddisfatta la seconda delle (8).

Infine, qualora fosse anche $a_{13} A_{43}+a_{23} A_{23}=0$, e quindi per la (4) $A_{33} \neq 0$, la (7) si riduce alla forma

$$
q^{3}-\bar{a}_{33} q^{2}+\left(\bar{A}_{11}+\bar{A}_{22}+A_{33}\right) q-\bar{a}_{33} A_{33}=0
$$

da cui

$$
a b-c=-\bar{a}_{33}\left(\bar{A}_{41}+\bar{A}_{22}\right) ;
$$

e poichè dalla (9), per la (5), in tal caso si ha $\bar{A}_{11}+\bar{A}_{22}<0$, basta scegliere $\bar{a}_{33} \neq 0$ perchè non risulti soddisfatta la terza delle (8).

Ciò completa la nostra verifica.

3. - Sia $\bar{a}_{33}$ un numero reale tale da garantire, per le considerazioni del numero precedente, la non esistenza di soluzioni periodiche non nulle del sistema (6), e posto $\rho=a_{33}-\bar{a}_{33}$, si consideri il sistema

$$
\left\{\begin{array}{l}
\dot{x}=a_{11} x+a_{12} y+a_{13} z \\
\dot{y}=a_{21} x+a_{22} y+a_{23} z \\
\dot{z}=a_{31} x+a_{32} y+\overline{a_{33} z}+\lambda[p(t, x, y, z)+f(z)+p z]
\end{array}\right.
$$

ove $\lambda$ ¿̀ un parametro reale che supponiamo variabile nell'intervallo chiuso $[0,1]$.

Tale sistema per $\lambda=0$ si riduce al sistema (6), mentre per $\lambda=1$ coin. cide col sistema (1).

Fissato $\lambda$ nell'intervallo $[0,1]$, sia $x=u(t), y=v(t), z=w(t)$ una (even- 
tuale) soluzione del corrispondente sistema (10), periodica di periodo 0 . Dalle prime due delle (10), tenendo conto della periodicità delle funzioni $u(t)$, $v(t), w(t)$, si ottiene con semplici calcoli il sistema algebrico

$$
\left\{\begin{array}{c}
a_{12} \int_{0}^{\omega} u(t) v(t) d t+a_{13} \int_{0}^{\omega} u(t) v(t) d t=-a_{11} \int_{0}^{\omega} u^{2}(t) d t \\
a_{21} \int_{0}^{\omega} u(t) v(t) d t+a_{23} \int_{0}^{\omega} v(t) w(t) d t=-a_{2} \cdot \int_{0}^{\omega} v^{2}(t) d t \\
\left(a_{11}+a_{22}\right) \int_{0}^{\omega} u(t) v(t) d t+a_{23} \int_{0}^{\omega} u(t) v(t) d t+a_{13} \int_{0}^{\omega} v(t) v(t) d t= \\
=-a_{21} \int_{0}^{\omega} u^{2}(t) d t-a_{12} \int_{0}^{\omega} v^{2}(t) d t
\end{array}\right.
$$

e da questa, supposta soddisfatta la condizione

$$
H=\alpha_{13} A_{32}+\alpha_{23} A_{31} \neq 0
$$

si ricavano le relazioni

$$
\begin{aligned}
& H \int_{0}^{\omega} u(t) v(t) d t=a_{23} A_{32} \int_{0}^{\omega} u^{2}(t) d t+a_{13} A_{31} \int_{0}^{\omega} v^{2}(t) d t \\
& H \int_{0}^{\omega} u(t) w(t) d t=\left(\alpha_{23} A_{33}-a_{31} A_{32}\right) \int_{0}^{\omega} u^{2}(t) d t-a_{12} A_{31} \int_{0}^{\omega} v^{2}(t) d t \\
& H \int_{0}^{\omega} v(t) w(t) d t=-a_{21} A_{32} \int_{0}^{\omega} u^{2}(t) d t+ \\
& \quad+\left(a_{13} A_{33}-a_{22} A_{31}\right) \int_{0}^{\omega} v^{2}(t) d t .
\end{aligned}
$$


Infine, dalla terza delle (10) si ottiene

$$
\begin{aligned}
\int_{0}^{\omega} \dot{w}^{2}(t) d t+a_{31} \int_{0}^{\omega} \dot{u}(t) w(t) d t & +a_{32} \int_{0}^{\omega} \dot{v}(t) w(t) d t= \\
& =\lambda \int_{0}^{\omega} \dot{w}(t) p[t, u(t), v(t), w(t)] d t
\end{aligned}
$$

ovvero

$$
\begin{aligned}
& H \int_{0}^{\omega} w^{2}(t) d t+\left(a_{11} a_{31}+a_{21} a_{32}\right) H \int_{0}^{\omega} u(t) w(t) d t+ \\
& +\left(a_{12} a_{31}+a_{22} a_{32}\right) H \int_{0}^{\omega} v(t) w(t) d t+ \\
& +\left(a_{13} a_{31}+a_{23} a_{32}\right) H \int_{0}^{\omega} w^{2}(t) d t=H \lambda \int_{0}^{\omega} \dot{w}(t) p[t, u(t), v(t), w(t)] d t
\end{aligned}
$$

e sostituendo in questa le due ultime delle (12):

$$
\begin{gathered}
\alpha \int_{0}^{\omega} u^{2}(t) d t+\beta \int_{0}^{\omega} v^{2}(t) d t+\left(a_{13} a_{31}+a_{33} a_{32}\right) H \int^{\omega} w^{2}(t) d t+ \\
+H \int_{0}^{\omega} \dot{w}^{2}(t) d t=H \lambda \int_{0}^{\omega} \dot{w}(t) p[t, u(t), v(t), w(t)] d t
\end{gathered}
$$

con

$$
\left\{\begin{array}{l}
\alpha=\left(a_{11} a_{31}+a_{21} a_{32}\right)\left(a_{23} A_{33}-a_{11} A_{32}\right)-\left(a_{12} a_{31}+a_{22} a_{32}\right) a_{21} A_{32} . \\
\beta=\left(a_{12} a_{31}+a_{22} a_{32}\right)\left(a_{18} A_{33}-a_{22} A_{31}\right)-\left(a_{11} a_{34}+a_{21} a_{32}\right) a_{12} A_{31} .
\end{array}\right.
$$

Supporremo soddisfatte le condizioni

$$
\alpha H \geq 0, \quad \beta H \geq 0 ;
$$

dalla (13), per la (5), la (11) e per la disuguaglianza di BUNIKowSKI-ScHwARZ 
(cfr. [4], p. 4), si ha allora

$$
\begin{aligned}
\int_{0}^{\omega} \dot{w}^{2}(t) d t \leq & \int_{0}^{\omega}|\dot{w}(t)| \mid p(t, u(t), v(t), w(t) \mid d t \leq \\
& \leq\left(\int_{0}^{\omega} p^{2} d t\right)^{1 / 2}\left(\int_{0}^{\omega} \dot{w}^{2}(t) d t\right)^{1 / 2}
\end{aligned}
$$

da cui

$$
\int_{0}^{\omega} \dot{w}^{2}(t) d t \leq p^{2} \omega, \quad\left(a_{13} a_{31}+a_{23} a_{32}\right) \int_{0}^{\omega} w^{2}(t) d t \leq p^{2} \omega .
$$

Ne segue (cfr. [6], p. 184) una limitazione per $|w(t)|$, e può dunque concludersi che: nelle ipotesi dichiarate, le eventuali soluzioni periodiche di periodo $\omega$ del sistema (10) hanno, al variare di $\lambda$, la terza componente mag. giorata in valore assoluto da una costante che non dipende da $\lambda$.

4. - Le conclusioni del numero precedente consentono di applicare allo studio dell'esistenza di soluzioni periodiche di sistemi del tipo (1) nn procedimento dimostrativo già adottato (cfr. [6]) per analogo problema nel caso di particolari classi di equazioni del terzo ordine.

Indichiamo con $\mathrm{Q}$ lo spazio dei vettori $\xi(t)=\left(\begin{array}{c}x(t) \\ y(t) \\ z(t)\end{array}\right)$ periodici di periodo $\omega$, con la norma definita da

$$
|\xi|=\max _{0 \leq i \leq \omega}\|\xi(t)\|, \quad\left[\|\xi(t)\|=\left(x^{2}(t)+y^{2}(t)+z^{2}(t)\right)^{3 / 2}\right]
$$

Sia $Y(t)$ la matrice fondamentale del sistema (6) individuata dalla condi. zione $Y(0)=I$ ( $I$ matrice $3 \times 3$ unita $)$, e sia

$$
F(t, \xi)=\left(\begin{array}{c}
0 \\
0 \\
p(t, x, y, z)+f(z)+\rho z
\end{array}\right)
$$

ove le funzioni $p$ ed $f$ e la costante $p$ sono quelle, precedentemente definite, che figurano nella terza equazione del sistema (10).

Ciò posto, ricordando che con opportuna scelta del numero $\overline{a_{33}}$ si garantisce la non risonanza del sistema (6) vale a dire la non esistenza di soluzioni periodiche non nulle, in particolare di periodo $\omega$, definiamo in $\Omega$ la 
trasformazione

$$
T_{\lambda,(\xi)}=\lambda Y(t)[I-Y(\omega)]^{-3} Y(\omega) \int_{t}^{t+\omega} Y^{-1}(\tau) F[\tau, \xi(\tau)] d \tau
$$

in cui $\lambda$ è un parametro reale che supporremo variabile nell' intervallo $[0,1]$. È noto (efr. [6], p. 183) che la (16) è una trasformazione completamente continua su $\Omega$; essa dipende con continuità dal parametro $\lambda$, e per $\lambda=0$ trasforma ogni elemento di $\Omega$ nel suo elemento neutro; inoltre, fissato $\lambda$ nel. l'intervallo considerato, gli eventuali elementi uniti della (16), cioè le soluzioni dell' equazione

$$
T_{\lambda}(\xi)-\xi=0
$$

sono tutte e sole le soluzioni periodiche di periodo $\omega$ del corrispondente sistema (10).

Ne segue, in virtù della (2) e delle conclusioni del numero precedente, che per $\lambda$ variabile nell'intervallo $[0,1]$ le eventuali soluzioni della (17) risultano ugualmente limitate in norma; e da qui, per un classico risultato di LERAY e SCHAdDER, si ottiene l'esistenza di un vettore $\xi(t)$ soddisfacente la condizione

$$
\xi(t)=Y(t)[I-Y(\omega)]^{-x} Y(\omega) \int_{t}^{t+\omega} Y^{-1}(\tau) F[\tau, \xi(\tau)] d \tau
$$

vale a dire l'esistenza di una soluzione periodica di periodo $\omega$ del sistema (1).

In conclusione si ha il

Teorema: Il sistema (1) presenta almeno una soluzione periodica di periodo w se il sistema omogeneo ad esso associato soddisfa le ipotesi (4), (5), (11) e (15), e se $p(t, x, y, z)$ e $f(z)$ sono funzioni continue dei rispettivi argomenti e $p(t, x, y, z)$ verifica le condizioni (2) e (3).

5. - È quasi superflno osservare che, scambiando nel sistema (1) l'ordine delle equazioni e delle componenti del vettore incognito, e riportando le ipotesi precedenti al caso dei sistemi

$$
\left\{\begin{array}{l}
\dot{x}=a_{11} x+a_{12} y+a_{13} z+p(t, x, y, z)+f(x), \\
\dot{y}=a_{21} x+a_{22} y+a_{23} z \\
\dot{z}=a_{31} x+a_{32} y+a_{33} z
\end{array}\right.
$$




$$
\left\{\begin{array}{l}
\dot{x}=a_{11} x+a_{12} y+a_{13} z \\
\dot{y}=a_{21} x+a_{32} y+a_{23} z+p(t, x, y, z)+f(y) \\
\dot{z}=a_{31} x+a_{32} y+a_{33} z
\end{array}\right.
$$

si ottengono risultati analoghi a quello contenuto nel Teorema precedente.

Tale Teorema esprime in sostanza l'attitudine a conservare le soluzioni periodiche di certi sistemi lineari (ad es., nel nostro caso, quelli a coefficienti costanti soddisfacenti condizioni come le (4), (5), (11) e (15)) quando siano soggetti a perturbazioni continue che interessano una sola equazione del sistema e possono decomporsi nella somma di una parte limitata e periodica $\Theta$ di una dipendente dalla sola componente che appare come derivata nel primo membro dell' equazione perturbata.

Ciò può essere confrontato con l'analoga proprietà che presentano i sistemi periodici, in condizioni di non risonanza, nei confronti di «piccole» perturbazioni continue (cfr. [6], p. 181); e va notato, a tale proposito, che, se $A_{33} \neq 0$, il Teorema precedente pnò applicarsi anche a sistemi (1) in cui si abbia $\operatorname{det} A=0$, e che presentano quindi il caso di risonanza.

Si osservi infine che, per l'equazione

$$
\ddot{x}=a_{33} \ddot{x}+a_{32} \dot{x}+a_{31} x+p(t, x, \dot{x}, \ddot{x})+f(\ddot{x}),
$$

ciò̀ per i sistemi (1) in cui $a_{12}=a_{23}=1, a_{11}=a_{13}=a_{21}=a_{22}=0$, si ha

$$
\begin{aligned}
& a_{13} A_{43}+a_{23} A_{23}=a_{31}, \\
& a_{43} a_{34}+a_{23} a_{32} \equiv a_{32}, \\
& H=1, \quad \alpha=\beta=0 ;
\end{aligned}
$$

le condizioni del Teorema si traducono quindi in: $a_{31} \neq 0, a_{32}>0$, e si ritrova un criterio di esistenza di soluzioni periodiche precedentemente stabilito (cfr. [6], n. 11; efr. anche [5]).

\section{BIBLIOGRAFIA}

[1] E. S. Anitova, Sulla limitateza delle soluzioni di un sistema del terzo ordine di equazioni differenziali (in rasso), Vestnik Leningradskozo Universiteta, 1964, n. 1, Matematika, pp. $5 \cdot 14$.

[2] - -, (stesso titolo), ibid., 1964, n. 7, Brevi Note, 149.151. 
[3] V. A. PuIss, Alcuni problemi nella teoria della stabilità in grande, (in russo), Ediz. Univ. Leningrado, 1958.

[4] G. SAnsone, Modena teoria delle funzioni di variabile reale, (Parte II), $2^{\mathrm{a}}$ ediz., Bologna. 1946.

[5] G. VILLARI, Criteri di esistenza di solwzioni periodiche per particolari classi di equa. zioni differenziali del ter's' ordine non lineari, Le Matematiche, 19 (1964), pp. 70-86.

$[6]-\ldots$, Contributi allo studio dell'esistenza di soluzioni periodiche per $i$ sistemi di equa. zioni differenziali ordinarie, questo volume, pp. 171-190. 